\begin{tabular}{|c|l|}
\hline Title & Imaging gigahertz surface acoustic waves through the photoelastic effect \\
\hline Author(s) & Saito, Taiki; Matsuda, Osamu; Tomoda, Motonobu; Wright, Oliver B. \\
\hline Citation & Journal of the Optical Society of A merica B: Optical Phy sics, 27(12), 2632-2638 \\
\hline https://doi.org/_0.1364/JOSA B.27.002632 \\
\hline Issue Date & 2010-12-01 \\
\hline Doc URL & http://hdl.handle.net/2115/44495 \\
\hline Rights & ○ 2010 Optical Society of A merica \\
\hline Type & article \\
\hline File Information & JOSA B27-12_2632-2638.pdf \\
\hline
\end{tabular}

Instructions for use 


\title{
Imaging gigahertz surface acoustic waves through the photoelastic effect
}

\author{
Taiki Saito, Osamu Matsuda,* Motonobu Tomoda, and Oliver B. Wright \\ Division of Applied Physics, Faculty of Engineering, Hokkaido University, Sapporo, Hokkaido 060-8628, Japan \\ *Corresponding author: omatsuda@eng.hokudai.ac.jp
}

Received August 12, 2010; revised October 12, 2010; accepted October 13, 2010; posted October 13, 2010 (Doc. ID 133294); published November 11, 2010

\begin{abstract}
This paper presents experiments and in-depth analysis of the imaging of surface acoustic waves by means of the photoelastic effect. Gigahertz surface acoustic waves, generated by optical pump pulses in a thin gold film on a glass substrate, are imaged in the time domain by monitoring ultrafast changes in optical reflectivity. We demonstrate how images of the in-plane acoustic shear strain component can be obtained by measurements with two different optical probe pulse polarizations incident from the substrate side. (C) 2010 Optical Society of America

OCIS codes: $110.5120,240.6690,260.1440,290.5825,110.7170,100.0118$
\end{abstract}

\section{INTRODUCTION}

A variety of optical techniques have been used to image electrically excited [1-7] or laser-excited [8-11] surface acoustic waves (SAWs) on solids and microstructures. When point-focused laser pulses are used for the SAW generation, the resulting omnidirectional twodimensional wave field can be mapped in the time domain and then Fourier-analyzed to obtain the in-plane acoustic dispersion relation [9,12-14]. This time-domain method, based on the optical pump-probe technique and interferometric detection, allows SAWs up to $\sim 1 \mathrm{GHz}$ to be imaged with micrometer spatial and picosecond temporal resolution [12]. Optical interferometry is convenient for such measurements because of its high (picometer-order) sensitivity to the surface displacement. However, this method suffers from the drawback of being only sensitive to the out-of-plane displacement component. Better knowledge of the strain field would be obtained by a measurement of the in-plane displacement as well.

One way to detect in-plane displacements is through the photoelastic effect by exploiting optical birefringence. This has been extensively used in the field of experimental mechanics to image static stress distributions in the bulk [15,16]. A typical experimental setup consists of a pair of crossed polarizers between which a transparent specimen is positioned, and one observes a pattern of fringes in optical transmission. This pattern is used to determine the stress distribution. In dynamic photoelasticity the temporal evolution of the stress field is imaged, including both longitudinal and shear acoustic wave propagation [17-21]. These techniques often involve samples having centimeter or larger dimensions with a temporal resolution down to $\sim \mu$ s. Optical phase retardations $>2 \pi$ are commonly obtained because the imaged stress is present over a significantly large portion the sample.

Similar techniques can be applied to detect stress (or strain) fields caused by SAWs traveling over regions of micrometer-order in size on nanosecond time scales. In this case, the phase retardation is very small compared to that in typical photoelasticity measurements. This requires measurement techniques and analysis somewhat different from those used in conventional photoelasticity experiments. For example, Miyamoto et al. observed shear-horizontal SAWs on an electrically driven $800 \mathrm{MHz}$ SAW filter device with a lateral spatial resolution of several micrometers [5]. Yamazaki et al. observed the acoustic field caused by point-like optical excitation through the photoelastic effect with micrometer spatial resolution and gigahertz bandwidth [22]. They observed a peculiar pattern on an isotropic thin film on an isotropic substrate, but the origin of the pattern was not quantitatively clarified. In this paper, we report on a gigahertz SAW imaging experiment based on the photoelastic effect and develop a theory to explain the observed pattern. We show how, by manipulating the optical polarization of the probe beam, one may isolate and image the in-plane acoustic shear strain field.

\section{EXPERIMENTAL SETUP AND RESULTS}

The sample is a crown glass plate of thickness $1 \mathrm{~mm}$ coated with a polycrystalline Au film of thickness $45 \mathrm{~nm}$ by radio-frequency sputtering. Figure 1 shows a schematic diagram of the experimental setup. A mode locked Ti:Sapphire laser with a center wavelength of $830 \mathrm{~nm}$, a repetition rate of $76 \mathrm{MHz}$, and a pulse duration of $150 \mathrm{fs}$ is used as a light source. The second harmonic (pump, wavelength of $415 \mathrm{~nm}$ ) is used for generating the acoustic waves, whereas the fundamental (probe, wavelength of $830 \mathrm{~nm}$ ) is used for detection. The energies of the pump and probe beams are 0.3 and $0.06 \mathrm{~nJ} /$ pulse, respectively. The pump light beam is shaped by a spatial filter, and then focused to a spot diameter of $\sim 2 \mu \mathrm{m}$ onto the sample at normal incidence with a $50 \times$ microscope objective from the $\mathrm{Au}$ film side of the sample. This excites broadband 


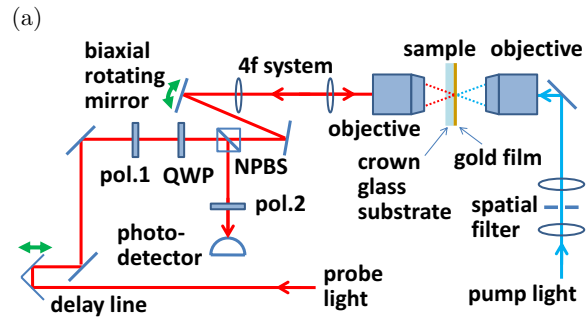

(b)

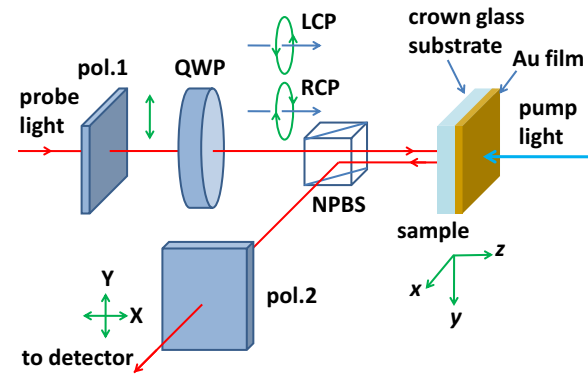

Fig. 1. (Color online) (a) Schematic diagram of the experimental setup for SAW imaging through the photoelastic effect: pol., polarizer; QWP, quarter wave plate; NPBS, non-polarizing beam splitter. (b) Polarization configurations for the measurement and the definitions of the polarizations $R, L, X$, and $Y$. The $x, y$, and $z$ axes are also shown. LCP and RCP, left and right circular polarization.

SAW pulses with frequency components up to $\sim 1 \mathrm{GHz}$, with a peak amplitude at $\sim 500 \mathrm{MHz}$. The probe light beam is focused to a spot diameter of $\sim 2 \mu \mathrm{m}$ on the sample from the substrate side with a second $50 \times$ microscope objective, allowing a lateral spatial resolution of the same order. In this study we measure the probe reflectivity modulation caused mainly by the strain in the glass substrate. (The photoelastic response of the $\mathrm{Au}$ film is negligible at the probe wavelength $[23,24]$.) We therefore expect a signal enhancement through the integration of the photoelastic response along the depth direction in the glass substrate. The delay time between pump and probe pulses is varied from 0 to $13.2 \mathrm{~ns}$ using a $0-4 \mathrm{~m}$ variable optical delay line. The probe light spot position is scanned over the sample surface in two dimensions using a twoaxis rotating mirror and a $4 f$ optical system [12]. The change in the intensity of the probe light reflected from the sample is recorded as a function of the delay time and the probe spot position. To improve the signal-to-noise ratio, the pump optical beam is modulated at $1 \mathrm{MHz}$, and the probe beam photodetector output is fed to a lock-in amplifier working at this frequency. Typical relative changes in reflectivity are $\sim 10^{-5}$ with a root-mean-square noise level of $\sim 10^{-6}$.

Figure 2 shows images of the optical intensity change $\Delta I$ at a time $11.7 \mathrm{~ns}$ after the pump light pulse arrival at the sample obtained with right (clockwise, viewed from the downstream direction) and left (anti-clockwise) circularly polarized probe light incident on the sample. A polarizer is placed in front of the photodetector to pass the $X$ or $Y$ polarization component of the reflected light [see Fig. 1(b)]. We refer to the incident and reflected light polarizations, A and B respectively, using the suffix A-B for quantities such as $\Delta I$. (A is chosen as either $L$ or $R$ : left or right circular polarization; in addition, B may be either $X$ or $Y$ :

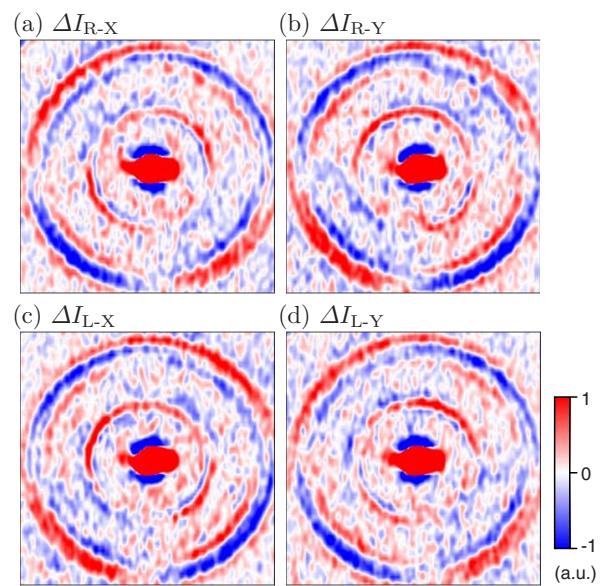

Fig. 2. (Color online) Images of the optical intensity change $\Delta I$ at a pump-probe delay time of $11.7 \mathrm{~ns}$ obtained with various polarization configurations for the probe light. The suffix notation A-B denotes the light polarization that is incident (A) and the polarization that is detected (B). A may be either $L$ or $R$ : left or right circular polarization. B may be either $X$ or $Y$ : linear polarization along $x$ or $y$ axis. The imaged area is $140 \mu \mathrm{m} \times 140 \mu \mathrm{m}$. The outer ring represents the SSLW and the inner ring represents the RW.

linear polarization along the $x$ or $y$ axis.) The imaged area is $140 \mu \mathrm{m} \times 140 \mu \mathrm{m}$. Concentric rings are clearly observed in Fig. 2. We estimate the phase velocity of the waves corresponding to the outer and inner rings in Fig. 2 as $\sim 5200$ and $2700 \mathrm{~m} / \mathrm{s}$. The literature values of the bulk longitudinal and Rayleigh wave (RW) velocities of crown glass are 5660 and $3130 \mathrm{~m} / \mathrm{s}$, respectively [25], so we identify these rings as surface skimming longitudinal waves (SSLWs) and RWs, respectively, with the differences being attributable to the presence of the $\mathrm{Au}$ film $[9,26]$. The waveforms depend on direction, giving rise to intriguing patterns, previously reported by Yamazaki et al. [22]. The pattern for each ring depends on the probe polarization configuration. The main aim of this paper is to understand the origin of these patterns.

\section{THEORY}

In order to quantitatively understand the experimental images, in this section we develop a theory for the reflection of a probe beam from an elastically perturbed medium. The reflectance change is caused by the inhomogeneous modulation of the permittivity, with contributions in general from both the photoelastic effect and interface displacements [27-31].

Consider a monochromatic probe beam with angular frequency $\omega$ and wave number $k=\omega / c$ in vacuum, where $c$ is the speed of light. Assuming a linear and quasi-static response for which the temporal variation of the permittivity is much slower than the optical frequency, the electric field amplitude variation $\boldsymbol{E}(\boldsymbol{r}, t)$ of the propagating probe light satisfies the equation

$$
\left(\nabla^{2}-\operatorname{grad} \operatorname{div}+k^{2} \varepsilon(\boldsymbol{r}, t)\right) \boldsymbol{E}(\boldsymbol{r}, t)=0,
$$

where $\varepsilon(\boldsymbol{r}, t)$ is the time and position dependent permittivity tensor, and the time dependence $\exp (-i \omega t)$ of the electric fields has been omitted. Hereafter the slower time 
dependence in $\varepsilon(\boldsymbol{r}, t)$ and $\boldsymbol{E}(\boldsymbol{r}, t)$ will not be explicitly expressed.

We restrict our discussion to normal probe light incidence. The $z$ axis is taken along its propagation direction. Over the dimensions of the optical probe spot the sample is assumed to be homogeneous in the $x y$ plane both in the equilibrium and strained states, and so the local variation of the permittivity depends on $z$ (and $t$ ) only. In this case Eq. (1) is reduced to

$$
\left\{L(z)+k^{2} \varepsilon(z)\right\} \boldsymbol{E}(z)=0,
$$

where the differential operator $L(z)$ is defined as

$$
L(z) \equiv\left(\begin{array}{ccc}
\partial^{2} / \partial z^{2} & 0 & 0 \\
0 & \partial^{2} / \partial z^{2} & 0 \\
0 & 0 & 0
\end{array}\right)
$$

and $\boldsymbol{E}$ depends only on $z$ (and $t$ ).

Hereafter we consider a sample consisting of a semiinfinite isotropic transparent medium (0) with permittivity $\varepsilon^{(0)}$ and a semi-infinite isotropic opaque medium (1) with permittivity $\varepsilon^{(1)}$ contacting each other at $z=0$. In reality the sample used in the experiment consists of a 45 $\mathrm{nm}$ gold thin film evaporated on a $1 \mathrm{~mm}$ glass plate. However, the film is thick enough to be regarded in optical calculations as an infinite opaque medium. In addition, the glass plate is much thicker than the coherence length of the optical pulse $(\sim 30 \mu \mathrm{m})$, implying no interference from light multiply reflected at the substrate interfaces. The semi-infinite medium approximation is therefore appropriate.

The $z$ dependent permittivity $\varepsilon(z)$ is expressed as the sum of a homogeneous part $\varepsilon_{h}(z)$ and an inhomogeneous part $\varepsilon_{i h}(z)$ :

$$
\begin{gathered}
\varepsilon(z) \equiv \varepsilon_{h}(z)+\varepsilon_{i h}(z), \\
\varepsilon_{h}(z) \equiv\left\{\begin{array}{l}
\varepsilon^{(0)} \boldsymbol{I} \text { for } z<0 \\
\varepsilon^{(1)} \boldsymbol{I} \text { for } z>0,
\end{array}\right.
\end{gathered}
$$

where $I$ is the $3 \times 3$ identity matrix. The quantity $\varepsilon_{i h}$ is a sum of contributions $\varepsilon_{\text {pe }}$ from the photoelastic effect and $\varepsilon_{\text {disp }}$ from the interface displacement. Even for media that are isotropic in their unperturbed states, the induced permittivity modulation $\varepsilon_{i h}$ may in general be anisotropic, and should therefore be expressed as a second rank tensor.

In order to solve Eq. (2) for a given $\varepsilon$, we first obtain a general solution for the non-perturbed equation

$$
\left\{L(z)+k^{2} \varepsilon_{h}(z)\right\} \boldsymbol{E}_{0}(z)=0,
$$

and also a Green's function $\boldsymbol{G}\left(z, z^{\prime}\right)$ that satisfies

$$
\left\{L(z)+k^{2} \varepsilon_{h}(z)\right\} \boldsymbol{G}\left(z, z^{\prime}\right)=-\delta\left(z-z^{\prime}\right) \boldsymbol{I} .
$$

The solution of Eq. (2) is then given by

$$
\boldsymbol{E}=\boldsymbol{E}_{0}(z)+k^{2} \int_{-\infty}^{\infty} \boldsymbol{G}\left(z, z^{\prime}\right) \varepsilon_{i h}\left(z^{\prime}\right) \boldsymbol{E}\left(z^{\prime}\right) \mathrm{d} z^{\prime} .
$$

When $\varepsilon_{i h}$ is small compared to $\varepsilon_{h}$, this can be approximated to first order as

$$
\boldsymbol{E} \simeq \boldsymbol{E}_{0}(z)+k^{2} \int_{-\infty}^{\infty} \boldsymbol{G}\left(z, z^{\prime}\right) \varepsilon_{i h}\left(z^{\prime}\right) \boldsymbol{E}_{0}\left(z^{\prime}\right) \mathrm{d} z^{\prime}
$$

Note that $\boldsymbol{G}$ and $\varepsilon_{i h}$ are non-commutative $3 \times 3$ matrices.

The solution of Eq. (8) should satisfy the electromagnetic boundary conditions for probe light incident from the transparent side. This leads to the following specific form for $\boldsymbol{E}_{0}$ :

$$
\begin{aligned}
& \boldsymbol{E}_{0}= \begin{cases}\left(e^{i k^{(0)} z}+r_{0} e^{-i k^{(0)}} z\right)\left(\begin{array}{c}
E_{x 0} \\
E_{y 0} \\
0
\end{array}\right) & \text { for } z<0 \\
t_{0} e^{i k^{(1)} z}\left(\begin{array}{c}
E_{x 0} \\
E_{y 0} \\
0
\end{array}\right) & \text { for } z>0,\end{cases} \\
& r_{0} \equiv \frac{k^{(0)}-k^{(1)}}{k^{(0)}+k^{(1)}}, \quad t_{0} \equiv \frac{2 k^{(0)}}{k^{(0)}+k^{(1)}}
\end{aligned}
$$

where $k^{(0)} \equiv \sqrt{\varepsilon^{(0)}} k$ and $k^{(1)} \equiv \sqrt{\varepsilon^{(1)}} k$ are the wave numbers in media 0 and 1 , respectively, and $E_{x 0}$ and $E_{y 0}$ are the electric field amplitudes of the incident light. Here, $r_{0}$ and $t_{0}$ are, respectively, the reflectance and transmittance for the electric field amplitude. The reflectivity (for optical intensity) $R$ is given by $\left|r_{0}\right|^{2}$.

The Green's function $\boldsymbol{G}$ also depends on the sample structure and electromagnetic boundary conditions. The observation point $z$ in Eq. (8) is chosen in medium 0 to be far enough away from the region where the permittivity is modulated. In order to calculate the modulation in the reflected light, we require $\boldsymbol{G}\left(z, z^{\prime}\right)$ for $z<0$ and $z<z^{\prime}$. According to a procedure described elsewhere [31], $\boldsymbol{G}$ can be shown to be given by

$$
\begin{gathered}
\boldsymbol{G}\left(z, z^{\prime}\right)=g\left(z, z^{\prime}\right)\left(\begin{array}{ccc}
1 & 0 & 0 \\
0 & 1 & 0 \\
0 & 0 & 0
\end{array}\right), \\
g\left(z, z^{\prime}\right) \equiv \frac{i e^{-i k^{(0)} z}}{2 k^{(0)}} \times \begin{cases}e^{i k^{(0)} z^{\prime}}+r_{0} e^{-i k^{(0)} z^{\prime}} & \text { for } z^{\prime}<0 \\
t_{0} e^{i k^{(1)} z^{\prime}} & \text { for } z^{\prime}>0 .\end{cases}
\end{gathered}
$$

Substituting Eqs. (9) and (10) into Eq. (8), the electric field at the observation point can be written as

$$
\begin{aligned}
\boldsymbol{E}(z)= & \left(e^{i k^{(0)} z}+r_{0} e^{-i k^{(0)} z}\right)\left(\begin{array}{c}
E_{x 0} \\
E_{y 0} \\
0
\end{array}\right)+e^{-i k^{(0)} z}\left(\begin{array}{c}
E_{x 0} Z_{1}+E_{y 0} Z_{6} \\
E_{x 0} Z_{6}+E_{y 0} Z_{2} \\
0
\end{array}\right) \\
& +2 i k^{(0)} u_{z}(0) r_{0} e^{-i k^{(0)}} z\left(\begin{array}{c}
E_{x 0} \\
E_{y 0} \\
0
\end{array}\right)
\end{aligned}
$$




$$
\begin{aligned}
Z_{I} \equiv & \frac{i k^{2}}{2 k^{(0)}}\left\{\int_{z}^{0}\left(e^{i k^{(0)} z^{\prime}}+r_{0} e^{-i k^{(0)} z^{\prime}}\right)^{2} \varepsilon_{\mathrm{pe}, I}\left(z^{\prime}\right) \mathrm{d} z^{\prime}\right. \\
& \left.+\int_{0}^{\infty}\left(t_{0} e^{i k^{(1)} z^{\prime}}\right)^{2} \varepsilon_{\mathrm{pe}, I}\left(z^{\prime}\right) \mathrm{d} z^{\prime}\right\},
\end{aligned}
$$

where $I=1, \ldots, 6$ specifies a component of the tensor $\varepsilon_{\mathrm{pe}}$ in the abbreviated suffix notation $(1=x x, 2=y y, 3=z z, 4=y z$, $5=z x$, and $6=x y$ ). The first term in Eq. (11a) depends on both the incident and reflected light fields for the unperturbed sample. The second term is the photoelastic contribution. This contains an integration over depth of the permittivity modulation that we abbreviate using the notation $Z_{I}$ of Eq. (11b). The appearance of $E_{y 0}$ in the $x$ component of the electric field and vice versa is a result of the birefringence converting $x$-polarized incident light to $y$-polarized scattered light, and vice versa. $Z_{I}$ consists of two terms corresponding to the photoelastic effect in media 0 and 1. The third term in Eq. (11a) arises from the interface displacement $u_{z}(0)$ along the $z$ axis. This only modulates the phase of the reflected light and cannot be detected through reflectivity measurements as in the present investigation.

Equation (11a) can be used to calculate the relative reflectivity change $(\Delta R / R$, which is proportional to $\Delta I$ in the measurement) for a configuration which is specified by the polarization of the incident and analyzed (i.e., detected) probe light. Here we consider some representative polarization configurations:

(1) $E_{x 0}=1, E_{y 0}=0$ (linearly polarized light denoted as $X$ ) and detection without any analyzer (denoted as $U$ ):

$$
\left(\frac{\Delta R}{R}\right)_{X-U}=2 \operatorname{Re}\left(\frac{Z_{1}}{r_{0}}\right) .
$$

Terms quadratic to $Z_{I}$ are usually too small to be detected and are neglected hereafter.

(2) $E_{x 0}=0, E_{y 0}=1$ (linearly polarized light $Y$ ) and detected without any analyzer:

$$
\left(\frac{\Delta R}{R}\right)_{Y-U}=2 \operatorname{Re}\left(\frac{Z_{2}}{r_{0}}\right) .
$$

(3) $E_{x 0}=1, E_{y 0}=1$ (linearly polarized light at $45^{\circ}$ to $X$, denoted as 45) and detected as $X$-polarized light:

$$
\left(\frac{\Delta R}{R}\right)_{45-X}=2 \operatorname{Re}\left(\frac{Z_{1}+Z_{6}}{r_{0}}\right) .
$$

(4) $E_{x 0}=1, E_{y 0}=1$ (same as above) and detected as $Y$-polarized light:

$$
\left(\frac{\Delta R}{R}\right)_{45-Y}=2 \operatorname{Re}\left(\frac{Z_{6}+Z_{2}}{r_{0}}\right) .
$$

(5) $E_{x 0}=1, E_{y 0}=-1$ [linearly polarized light at $45^{\circ}$ to $X$ in the opposite direction to (3), denoted as $\overline{45}]$ and detected as $X$-polarized light:

$$
\left(\frac{\Delta R}{R}\right)_{\overline{45-X}}=2 \operatorname{Re}\left(\frac{Z_{1}-Z_{6}}{r_{0}}\right) .
$$

(6) $E_{x 0}=1, E_{y 0}=-1$ (same as above) and detected as $Y$-polarized light:

$$
\left(\frac{\Delta R}{R}\right)_{\overline{45-Y}}=2 \operatorname{Re}\left(\frac{Z_{2}-Z_{6}}{r_{0}}\right) .
$$

(7) $E_{x 0}=1, E_{y 0}=i$ (left circularly polarized light, denoted by $L$ ) and detected as $X$-polarized light:

$$
\left(\frac{\Delta R}{R}\right)_{L-X}=2 \operatorname{Re}\left(\frac{Z_{1}+i Z_{6}}{r_{0}}\right) .
$$

(8) $E_{x 0}=1, E_{y 0}=i$ (same as above) and detected as $Y$-polarized light:

$$
\left(\frac{\Delta R}{R}\right)_{L-Y}=2 \operatorname{Re}\left(\frac{Z_{2}-i Z_{6}}{r_{0}}\right) .
$$

(9) $E_{x 0}=1, E_{y 0}=-i$ (right circularly polarized light, denoted by $R$ ) and detected as $X$-polarized light:

$$
\left(\frac{\Delta R}{R}\right)_{R-X}=2 \operatorname{Re}\left(\frac{Z_{1}-i Z_{6}}{r_{0}}\right) .
$$

(10) $E_{x 0}=1, E_{y 0}=-i$ (same as above) and detected as $Y$-polarized light:

$$
\left(\frac{\Delta R}{R}\right)_{R-Y}=2 \operatorname{Re}\left(\frac{Z_{2}+i Z_{6}}{r_{0}}\right) .
$$

One can see that it is possible to extract $Z_{6}$ separately by subtracting independent measurements:

$$
\begin{aligned}
& 4 \operatorname{Re}\left(\frac{Z_{6}}{r_{0}}\right)=\left(\frac{\Delta R}{R}\right)_{45-X}-\left(\frac{\Delta R}{R}\right)_{\overline{45-X}} \\
&=\left(\frac{\Delta R}{R}\right)_{45-Y}-\left(\frac{\Delta R}{R}\right)_{\overline{45-Y}}, \\
& 4 \operatorname{Im}\left(\frac{Z_{6}}{r_{0}}\right)=\left(\frac{\Delta R}{R}\right)_{R-X}-\left(\frac{\Delta R}{R}\right)_{L-X}=\left(\frac{\Delta R}{R}\right)_{L-Y}-\left(\frac{\Delta R}{R}\right)_{R-Y} .
\end{aligned}
$$

Another interesting combination is a sum of independent measurements:

$$
\begin{aligned}
& 4 \operatorname{Re}\left(\frac{Z_{1}+Z_{2}}{r_{0}}\right)=\left(\frac{\Delta R}{R}\right)_{45-X}+\left(\frac{\Delta R}{R}\right)_{\overline{45}-Y} \\
&=\left(\frac{\Delta R}{R}\right)_{45-Y}+\left(\frac{\Delta R}{R}\right)_{\overline{45-X}} \\
&=\left(\frac{\Delta R}{R}\right)_{R-X}+\left(\frac{\Delta R}{R}\right)_{R-Y}=\left(\frac{\Delta R}{R}\right)_{L-X}+\left(\frac{\Delta R}{R}\right)_{L-Y} .
\end{aligned}
$$

To proceed further the explicit form of $\varepsilon_{i h}$ is required. For isotropic media, as in the present experiment, the photoelastic permittivity modulation is given by

$$
\varepsilon_{\mathrm{pe}, 1}=P_{11} \eta_{1}+P_{12}\left(\eta_{2}+\eta_{3}\right),
$$




$$
\begin{gathered}
\varepsilon_{\mathrm{pe}, 2}=P_{11} \eta_{2}+P_{12}\left(\eta_{3}+\eta_{1}\right), \\
\varepsilon_{\mathrm{pe}, 3}=P_{11} \eta_{3}+P_{12}\left(\eta_{1}+\eta_{2}\right), \\
\varepsilon_{\mathrm{pe}, I}=P_{44} \eta_{I} \quad(I=4,5,6),
\end{gathered}
$$

where $\eta_{I}$ and $P_{I J}$ denote components of the strain and photoelastic tensors, respectively, in the abbreviated suffix notation. Here we consider $\varepsilon_{\mathrm{pe}, I}$ and $\eta_{I}$ as functions of $z$. $P_{I J}$ is also a function of $z$, but it depends only on the medium in which the point $z$ resides. For an isotropic medium, $P_{44}=\left(P_{11}-P_{12}\right) / 2$. It turns out that $Z_{6}$ depends only on the shear strain component $\eta_{6}$, whereas $Z_{1}$ and $Z_{2}$ depend on longitudinal strain components $\eta_{1}, \eta_{2}$, and $\eta_{3}$ in a relatively complicated way.

We now consider acoustic waves generated at a point source in a laterally homogeneous isotropic medium and propagating along the surface of the medium, as in the present experiment. We make use of Cartesian coordinates $(x, y, z)$ and cylindrical coordinates $(r, \theta, z)$, with their origin $O$ at the source point (where $x=r \cos \theta$ and $y=r \sin \theta$ ). Using the unit vectors $\boldsymbol{e}_{x}, \boldsymbol{e}_{y}, \boldsymbol{e}_{z}$ and $\boldsymbol{e}_{r}, \boldsymbol{e}_{\theta}, \boldsymbol{e}_{z}$ for these coordinates, the acoustic displacement $\boldsymbol{u}$ is expressed as $\boldsymbol{u}=u_{x} \boldsymbol{e}_{x}+u_{y} \boldsymbol{e}_{y}+u_{z} \boldsymbol{e}_{z}=u_{r} \boldsymbol{e}_{r}+u_{\theta} \boldsymbol{e}_{\theta}+u_{z} \boldsymbol{e}_{z}$, where $\boldsymbol{e}_{r}=\cos \theta \boldsymbol{e}_{x}+\sin \theta \boldsymbol{e}_{y}, \boldsymbol{e}_{\theta}=-\sin \theta \boldsymbol{e}_{x}+\cos \theta \boldsymbol{e}_{y}$. Due to the symmetry of the system, $u_{\theta}$ is always zero, and $u_{r}$ and $u_{z}$ depend only on $r$ and $z$. The strain components in Cartesian coordinates can be expressed using displacement components in cylindrical coordinates:

$$
\begin{gathered}
\eta_{1}=\frac{\partial u_{r}}{\partial r} \cos ^{2} \theta+\frac{u_{r}}{r} \sin ^{2} \theta, \\
\eta_{2}=\frac{\partial u_{r}}{\partial r} \sin ^{2} \theta+\frac{u_{r}}{r} \cos ^{2} \theta, \\
\eta_{3}=\frac{\partial u_{z}}{\partial z}, \\
\eta_{5}=\left(\frac{\partial u_{r}}{\partial z}+\frac{\partial u_{z}}{\partial r}\right) \sin \theta, \\
\eta_{6}=2\left(\frac{\partial u_{r}}{\partial r}-\frac{\partial u_{z}}{\partial r}\right) \cos \theta, \cos \theta \sin \theta .
\end{gathered}
$$

Here, $\eta_{6}=\partial u_{y} / \partial x+\partial u_{x} / \partial y$ is zero along the $x$ and $y$ axes for our example of axisymmetric waves. The permittivity modulation relevant to our experiment is therefore given by

$$
\begin{aligned}
\varepsilon_{\mathrm{pe}, 1}= & \left(\frac{\partial u_{r}}{\partial r} P_{11}+\frac{u_{r}}{r} P_{12}\right) \cos ^{2} \theta+\left(\frac{\partial u_{r}}{\partial r} P_{12}+\frac{u_{r}}{r} P_{11}\right) \sin ^{2} \theta \\
& +\frac{\partial u_{z}}{\partial z} P_{12},
\end{aligned}
$$

$$
\begin{aligned}
\varepsilon_{\mathrm{pe}, 2}= & \left(\frac{\partial u_{r}}{\partial r} P_{11}+\frac{u_{r}}{r} P_{12}\right) \sin ^{2} \theta+\left(\frac{\partial u_{r}}{\partial r} P_{12}+\frac{u_{r}}{r} P_{11}\right) \cos ^{2} \theta \\
& +\frac{\partial u_{z}}{\partial z} P_{12},
\end{aligned}
$$$$
\varepsilon_{\mathrm{pe}, 6}=2\left(\frac{\partial u_{r}}{\partial r}-\frac{u_{r}}{r}\right) \cos \theta \sin \theta .
$$

From the expression for $\varepsilon_{\mathrm{pe}, 6}$ in Eq. (26), the images involving $Z_{6}$ obtained in Eq. (22) should exhibit a $\sin 2 \theta$ dependence. From the expressions for $\varepsilon_{\text {pe,1 }}$ and $\varepsilon_{\text {pe,2 }}$ in Eq. (26), the sum image of $Z_{1}$ and $Z_{2}$ obtained in Eq. (23) is related to

$$
\varepsilon_{\mathrm{pe}, 1}+\varepsilon_{\mathrm{pe}, 2}=\left(\frac{\partial u_{r}}{\partial r}+\frac{u_{r}}{r}\right)\left(P_{11}+P_{12}\right)+2 \frac{\partial u_{z}}{\partial z} P_{12} .
$$

There should thus be no $\theta$ dependence in this sum image. These results are discussed and compared to experiment in the next section.

\section{DISCUSSION}

Before definitively assigning the optical intensity change in experiment to the photoelastic effect, one should consider the possibility of a contribution from induced changes in beam divergence [22,32]. Beam divergence changes can be translated into detected intensity changes if apertures or irises are present in the detection optics. In the present experiment no irises are used to filter the probe beam after reflection from the sample; and, moreover, we make use of a $50 \times$ objective whose aperture is larger than the diameter of the probe beam. We therefore expect a negligible contribution from any modulation in beam divergence.

The glass substrate and Au film used are elastically isotropic and can be regarded as, respectively, transparent (0) and opaque (1) semi-infinite media. The theory presented in this paper is thus appropriate for data analysis.

Figure 3(a) shows the image obtained by subtracting Fig. 2(c) from Fig. 2(a). According to Eq. (22), this should be proportional to $Z_{6}$. From Eq. (26), the $Z_{6}$ image is expected to exhibit an angular dependence given by $\sin 2 \theta$. This is clearly observed in Fig. 3(a): both the rings due to the RWs and SSLWs show zero intensity for $\theta=m \pi / 2$ (where $m$ is an integer) and show maxima or minima in intensity for $\theta=(2 m+1) \pi / 4$.

Figure 3(b) shows an image that is obtained by subtracting Fig. 2(b) from Fig. 2(d). According to Eqs. (22) and (26), an image identical to Fig. 3(a) is expected. This is indeed observed to a good approximation, and so we conclude that this image originates solely from the shear strain component $\eta_{6}$ that governs the in-plane ultrasonic displacement.

Figure 3(c) shows the sum of Figs. 2(a) and 2(b), and Fig. 3(d) shows the sum of Figs. 2(c) and 2(d). Equation (27) predicts no $\theta$ dependence for these images. Residual variations with $\theta$ in these images are therefore attributed to ellipticity in the shape of the pump light spot, as evi- 


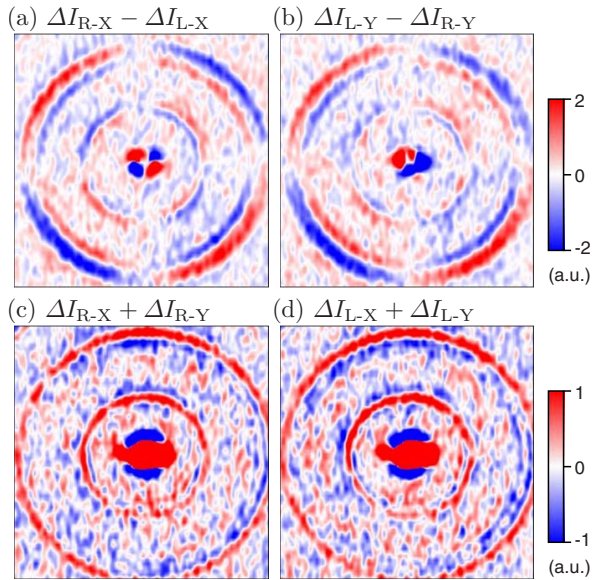

Fig. 3. (Color online) Images of the difference or sum of $\Delta I$ of Fig. 2. (a), (b) Difference images as defined at the top of each figure. These are related to the image of $\eta_{x y}$. (c), (d) Sum images. These are related to $\eta_{x x}, \eta_{y y}$, and $\eta_{z z}$. The imaged area is $140 \mu \mathrm{m} \times 140 \mu \mathrm{m}$ and the pump-probe delay time is $11.7 \mathrm{~ns}$. The outer ring represents the SSLW and the inner ring represents the RW.

denced by an elongation along the $x$ axis of the central thermally induced spot in Figs. 3(c) and 3(d).

On comparing the subtraction and sum images, the spatial extent of the SSLW wavepacket along the radial direction is broader in the former image than in the latter. It turns out that the RW wavepacket is more efficiently observed than the SSLW wavepacket in the sum images, and that the outer thin ring in Figs. 3(c) and 3(d) contain a dominant contribution from the second RW wavepacket (generated by the pump light pulse prior the inner RW wavepacket). This reduction in the SSLW intensity in the sum image is not understood at present. A detailed analysis would require three-dimensional modeling of the acoustic strain field and associated photoelastic response, which is beyond the scope of this paper.

Reproduction of the entire acoustic strain field from the experimental data would require solving the equation for $u_{r}:$

$$
\frac{\partial u_{r}}{\partial r}-\frac{u_{r}}{r}=f(r),
$$

with a given $f(r)$. The general solution for $u_{r}(r)$ is

$$
u_{r}(r)=r \int \frac{f(r)}{r} \mathrm{~d} r+C r,
$$

where $C$ is a constant. This implies that, if required, $u_{r}(r, z)$ could in theory be obtained from the experimental results provided that the $z$ dependence of the strain field could be measured by some other means [33-36].

\section{CONCLUSIONS}

In conclusion, we have obtained time-resolved images in two dimensions of surface acoustic wave (SAW) propagation on glass through transient optical reflectivity changes arising from the photoelastic effect. A theoretical treatment is presented that allows the data to be analyzed and allows an image that depends solely on the in- plane ultrasonic displacement to be extracted. This technique can detect small strain amplitudes $\left(\sim 10^{-5}\right)$ over microscopic depths $(<10 \mu \mathrm{m})$. In the future, it should also be possible to extend these experimental and theoretical methods to the analysis of anisotropic samples that exhibit more complicated acoustic and optical properties.

\section{ACKNOWLEDGMENT}

This work is partially supported by a Grant-in-Aid for Scientific Research from the Ministry of Education, Science, Sports, and Culture of Japan (MESSC-JP).

\section{REFERENCES AND NOTES}

1. R. Adler, A. Korpel, and P. Desmares, "An instrument for making surface waves visible," IEEE Trans. Ultrason. Ferroelectr. Freq. Control 15, 157-161 (1968).

2. J. V. Knuuttila, P. T. Tikka, and M. M. Salomaa, "Scanning Michelson interferometer for imaging surface acoustic wave fields," Opt. Lett. 25, 613-615 (2000).

3. J. E. Graebner, B. P. Barber, P. L. Gammel, and D. S. Greywall, "Dynamic visualization of subangstrom highfrequency surface vibrations," Appl. Phys. Lett. 78, 159161 (2001).

4. J. L. Blackshire, S. Sathish, B. D. Duncan, and M. Millard, "Real-time, frequency-translated holographic visualization of surface acoustic wave interactions with surface-breaking defects," Opt. Lett. 27, 1025-1027 (2002).

5. A. Miyamoto, S. Matsuda, S. Wakana, and A. Ito, "Optical observation technique for SH-type surface acoustic wave," Electron. Commun. Jpn., Part 2: Electron. 87, 1295-1301 (2004).

6. K. Kokkonen and M. Kaivola, "Scanning heterodyne laser interferometer for phase-sensitive absolute-amplitude measurements of surface vibrations," Appl. Phys. Lett. 92, 063502 (2008).

7. T. Fujikura, O. Matsuda, D. M. Profunser, O. B. Wright, J. Masson, and S. Ballandras, "Real-time imaging of acoustic waves on a bulk acoustic resonator," Appl. Phys. Lett. 93, 261101 (2008).

8. M. Clark, S. D. Sharples, and M. G. Somekh, "Diffractive acoustic elements for laser ultrasonics,” J. Acoust. Soc. Am. 107, 3179-3185 (2000).

9. Y. Sugawara, O. B. Wright, O. Matsuda, M. Takigahira, Y. Tanaka, S. Tamura, and V. E. Gusev, "Watching ripples on crystals," Phys. Rev. Lett. 88, 185504 (2002).

10. J. A. Scales and A. E. Malcolm, "Laser characterization of ultrasonic wave propagation in random media," Phys. Rev. E 67, 046618 (2003)

11. A. A. Maznev, A. M. Lomonosov, P. Hess, and A. A. Kolomenskii, "Anisotropic effects in surface acoustic wave propagation from a point source in a crystal," Eur. Phys. J. B 35, 429-439 (2003).

12. T. Tachizaki, T. Muroya, O. Matsuda, Y. Sugawara, D. H. Hurley, and O. B. Wright, "Scanning ultrafast sagnac interferometry for imaging two-dimensional surface wave propagation," Rev. Sci. Instrum. 77, 043713 (2006).

13. D. M. Profunser, O. B. Wright, and O. Matsuda, "Imaging ripples on phononic crystals reveals acoustic band structure and Bloch harmonics," Phys. Rev. Lett. 97, 055502 (2006).

14. D. M. Profunser, E. Muramoto, O. Matsuda, and O. B. Wright, "Dynamic visualization of surface acoustic waves on a two-dimensional phononic crystal," Phys. Rev. B 80, 014301 (2009).

15. M. M. Frocht, Photoelasticity (Wiley, 1957), Vols. 1 and 2.

16. C. P. Burger, "Photoelasticity," in Handbook on Experimental Mechanics (Second Revised Edition), A. S. Kobayashi, ed. (VCH, 1993), Chap. 5, pp. 165-266.

17. W. F. Riley and J. W. Dally, "A photoelastic analysis of stress wave propagation in a layered model," Geophysics 31, 881-899 (1966). 
18. J. W. Dally, "An introduction to dynamic photoelasticity," Exp. Mech. 20, 409-416 (1980).

19. Y. H. Nam and S. S. Lee, "A quantitative evaluation of elastic wave in solid by strobscopic photoelasticity," J. Sound Vib. 259, 1199-1207 (2003).

20. W.-C. Wang and Y.-H. Tsai, "Digital dynamic photoelastic and numerical stress analyses of a strip," J. Vib. Control 12 , 927-938 (2006)

21. R. Hayasi, Y. Masuda, S. Hashimoto, and S. Kuriyama, "Analysis of geometric effects on stress wave propagation in epoxy resins of plate-like structure by dynamic photoelasticity combined with strain gauge,” Jpn. J. Appl. Phys. 47, 4676-4681 (2008).

22. H. Yamazaki, O. Matsuda, and O. B. Wright, "Surface phonon imaging through the photoelastic effect," Phys. Status Solidi C 1, 2991-2994 (2004).

23. M. Garfinkel, J. J. Tiemann, and W. E. Engeler, "Piezoreflectivity of the noble metals," Phys. Rev. 148, 695-706 (1966).

24. This is supported by measurements made by probing from the film side of our sample, showing a response $\sim 10$ times smaller. Other measurements [35] also indicate a negligible photoelastic coefficient $P_{12}$ at our probe wavelength. The photoelastic constant of fused silica in the visible at this wavelength is approximately $P_{12}=-1.3$ [36].

25. G. W. C. Kaye and T. H. Laby, Tables of Physical and Chemical Constants, 16th ed. (Longman, 1995).

26. The SSLW ring in fact overlaps in Fig. 2 with a weaker second RW ring, but the former ring dominates.

27. C. Thomsen, H. T. Grahn, H. J. Maris, and J. Tauc, "Surface generation and detection of phonons by picosecond light pulses," Phys. Rev. B 34, 4129-4138 (1986).

28. B. Perrin, B. Bonello, J. C. Jeannet, and E. Romatet, "Interferometric detection of hypersound waves in modulated structures,” Prog. Nat. Sci. S6, S444-S448 (1996).

29. V. E. Gusev, "Laser hypersonics in fundamental and applied research," Acust. Acta Acust. 82, S37-S45 (1996).

30. O. Matsuda and O. B. Wright, "Reflection and transmission of light in multilayers perturbed by picosecond strain pulse propagation," J. Opt. Soc. Am. B 19, 3028-3041 (2002).

31. O. Matsuda, O. B. Wright, D. H. Hurley, V. E. Gusev, and K. Shimizu, "Coherent shear phonon generation and detection with picosecond laser acoustics," Phys. Rev. B 77, 224110 (2008).

32. N. Chigarev, C. Rossignol, and B. Audoin, "Surface displacement measured by beam distortion detection technique: Application to picosecond ultrasonics," Rev. Sci. Instrum. 77, 114901 (2006)

33. C. Matteï, X. Jia, and G. Quentin, "Measurement of Rayleigh wave strains inside a transparent solid by optical interferometry," Acta Acust. 2, 65-67 (1994).

34. M. Tomoda, O. Matsuda, and O. B. Wright, "Tomographic reconstruction of picosecond acoustic strain propagation," Appl. Phys. Lett. 90, 041114 (2007).

35. D. H. Hurley and O. B. Wright, "Detection of ultrafast phenomena by use of a modified sagnac interferometer," Opt. Lett. 24, 1305-1307 (1999).

36. R. W. Dixon, "Photoelastic properties of selected materials and their relevance for applications to acoustic light modulators and scanners,” J. Appl. Phys. 38, 5149-5153 (1967). 